\title{
Preoperative Surveillance Thoracic MRI for Thoracic Dorsal Column Stimulation: Case Series
}

\author{
Alexios Carayannopoulos, DOํ, Alex Han, BS¹, Albert E. Telfeian, MD, PhD², and Keith Scarfo, DO
}

Background: To date, there have been no guidelines, studies, or consensus statements regarding the use of advanced imaging studies as a preoperative screening tool prior to patients undergoing spinal cord stimulation (SCS).

Objectives: The purpose of this case series is to highlight the importance of obtaining thoracic imaging studies as part of a thorough initial clinical assessment prior to percutaneously or surgically placing an SCS electrode.

Methods: We undertook a 3-year retrospective review of patients undergoing evaluation for SCS therapy in an academic interventional pain management and neurosurgery practice. Patients with chronic neuropathic pain underwent a trial with percutaneous dorsal column stimulation, and if response to the trial was $>50 \%$ reduction in pain and improvement in function, the patient was implanted using either a percutaneous approach or placement of a paddle electrode with an internal pulse generator (IPG). All patients had advanced thoracic imaging performed (magnetic resonance imaging $[\mathrm{MRI}]$ if possible, or computed tomography [CT] if contraindicated) prior to percutaneous trial or paddle electrode implantation.

Results: In 3 years, 88 patients underwent a trial of SCS, of which 75 patients ( $85 \%$ ) were referred for implantation of a permanent percutaneous or paddle stimulator with IPG. Two patients (1.2\%) treated by trial and implant were found to have a thoracic lesion on surveillance imaging, which contraindicated SCS implantation. One patient had thoracic imaging prior to undergoing the trial and one patient had thoracic surveillance imaging after the trial, but before surgical paddle placement.

Limitations: The retrospective nature and lack of a control group in this case series are limitations of this study.

Conclusion: This case series outlines the importance of considering advanced thoracic imaging studies for screening/surveillance purposes prior to SCS therapy. Placement of SCS electrodes in the thoracic epidural space without prior understanding of spinal anatomy potentially puts patients at unnecessary risk. In the cases presented, had patients undergone SCS trial and subsequent implant without screening imaging, thoracic lesions would have been missed, increasing the likelihood of diagnostic and therapeutic complications. Our incidental findings of lesions on MRI prior to SCS trial or implant support the use of a screening protocol for other centers providing thoracic SCS therapies.

Key words: Thoracic, dorsal column stimulation, chronic pain, surveillance MRI, patient selection
From: ${ }^{1}$ Department of Physical Medicine and Rehabilitation, Rhode Island Hospital, The Warren Alpert Medical School of Brown University, Providence, RI; ${ }^{2}$ Department of Neurosurgery, Rhode Island Hospital, The Warren Alpert Medical School of Brown University, Providence, RI

Author for correspondence: Alexios Carayannopoulos, DO Address: Comprehensive Spine Center, Rhode Island Hospital, 593 Eddy St., Providence, RI 02903

E-mail: Acarayannopoulos@Lifespan.org
Spinal cord stimulation (SCS) is a continuously evolving technology and is commonly used to treat chronic, intractable neuropathic pain syndromes of the trunk and/or limbs, which include post-laminectomy syndrome, chronic regional pain syndrome, and others (1). As the field of neuromodulation continues to expand its indications and refine its applications, ensuring patient safety and suitability 
for SCS implantation should remain paramount (2). In this case series, we seek to illustrate how the use of preoperative thoracic imaging as a screening tool can help avert surgical complications, which in turn will allow providers to more appropriately triage and select patients for SCS therapy in the future.

\section{METHODS}

This is a retrospective, observational review of 163 consecutive patients who underwent evaluation for SCS over a 3-year period between July 2014 and July 2017 at Rhode Island Hospital. These included patients who were trialed and implanted by the same physician, as well as patients who were implanted after referral from an outside-hospital trialing physician. Indications for SCS included post-laminectomy pain syndrome, chronic regional pain syndrome, and other neuropathic pain syndromes. Prior to being considered for implantation, a stepwise multidisciplinary approach including conservative medical management, psychological counseling, physical and/or occupational therapy, as well as complementary and alternative medical treatments were attempted. Patients found to be candidates for SCS subsequently underwent an outpatient trial with an external SCS device to determine clinical efficacy. A trial was considered successful if it achieved a benchmark of $>50 \%$ pain relief as well as objective improvement in baseline functioning (3) prior to either percutaneous or surgical paddle electrode placement, which depended on the practice pattern of the interventional pain physician and/or neurosurgeon. No patients had surgical paddle placement without advanced thoracic imaging.

\section{RESULTS}

Case 1. Patient is a 58-year-old male presenting with low back and bilateral lower extremity pain and paresthesias, distal more than proximal, with associated numbness and loss of balance, leading to falls. An initial trial of physical therapy (PT) failed and subsequent lumbar magnetic resonance imaging (MRI) revealed mild central and foraminal stenosis. After a trial of epidural steroid injections and adjuvant neuropathic pain medications failed, he then underwent decompressive lumbar laminectomy surgery with bilateral foraminotomy.
The patient's leg pain improved marginally for one month before symptoms recurred. Repeat MRI with contrast showed persistent L4-L5 foraminal narrowing, mildly enhancing scar tissue, and persistent multilevel neural impingement. He then underwent additional surgery including repeat laminectomy at L4-L5, with subsequent posterior lumbar interbody fusion (PLIF) from L4-S1. Back and leg pain improved for 3 months, but despite repeat surgical intervention, the patient was impaired by progressive numbness and weakness. Additionally, he experienced episodes of bladder urgency, which, upon recollection, had been present for years.

He was then evaluated by Urology and started on medication to control bladder symptoms, thought to be secondary to mild benign prostatic hypertrophy. He was reevaluated by a spine surgeon who sent him for pain management. The patient's symptoms were unsuccessfully treated with bilateral transforaminal injections at L4-L5, non-opioid medication, and additional physical therapy, and he was subsequently referred for consideration of a spinal cord stimulator trial.

Upon evaluation, the patient was found to be an appropriate and motivated candidate with realistic expectations for SCS therapy and underwent routine medical and radiological screening, including limited noncontrast thoracic MRI. Initial thoracic MRI revealed an incidental finding of a cystic intra-axial lesion within the cord at T9. Follow-up contrast study revealed a non-enhancing, expansile, intramedullary, intradural, cystic, T2 bright cord lesion, with associated syrinx, and cord edema above and below the lesion (Fig. 1A). Computed tomography (CT) with myelogram showed significant narrowing at the level of T9 consistent with an extrinsic extramedullary arachnoid cyst with dilatation of the spinal cord consistent with a syrinx in the arachnoid space, without clear septations.

The patient was subsequently referred to a neurosurgeon for surgical excision of the lesion. It was believed that residual lower-extremity symptoms were related to the radiographic abnormality discovered on thoracic MRI. In fact, the lesion was deemed the likely cause of his original right leg numbness, bladder issues, and lack of resolution with initial surgical 
intervention. Given progression of numbness and weakness, exploration at the T9 level was pursued to explore the cystic lesion, with potential treatment of the syrinx itself.

The patient underwent thoracic laminectomy with fenestration of the arachnoid cyst, which had formed from thickened and opacified arachnoid, at the level of greatest spinal cord compression. The arachnoid tissue was widely resected and fenestration of the arachnoid cyst was performed to resolve the intramedullary cyst over time (Fig. 1B).

Following surgical management, the patient experienced a gradual reduction in proximal pain and numbness with improvement in ambulation, still requiring a gait aid for ambulation. The patient continued to describe a cramping sensation, pain, and leg weakness.
Follow-up thoracic MRI with contrast revealed a stable T2 bright signal at T8-9 with significant improvement in swelling status post resection of the arachnoid cyst closely adhered to the thoracic cord, with evidence of wide dorsal laminectomy at T8 through T11. There was no evidence of recurrent cyst. Follow-up MRI of the lumbar spine with and without contrast showed enhancing scar tissue at L4 and L5 from previous surgical intervention, without neural impingement.

Due to persistent, functionally limiting distal leg pain and numbness, the patient agreed to undergo a 3-day permanent paddle trial with the top of the electrode surgically placed at T8 by a neurosurgeon. During the trial, the patient experienced greater than $50 \%$ improvement in pain and significant improvement in function, so he elected to proceed forward with a permanent implant.
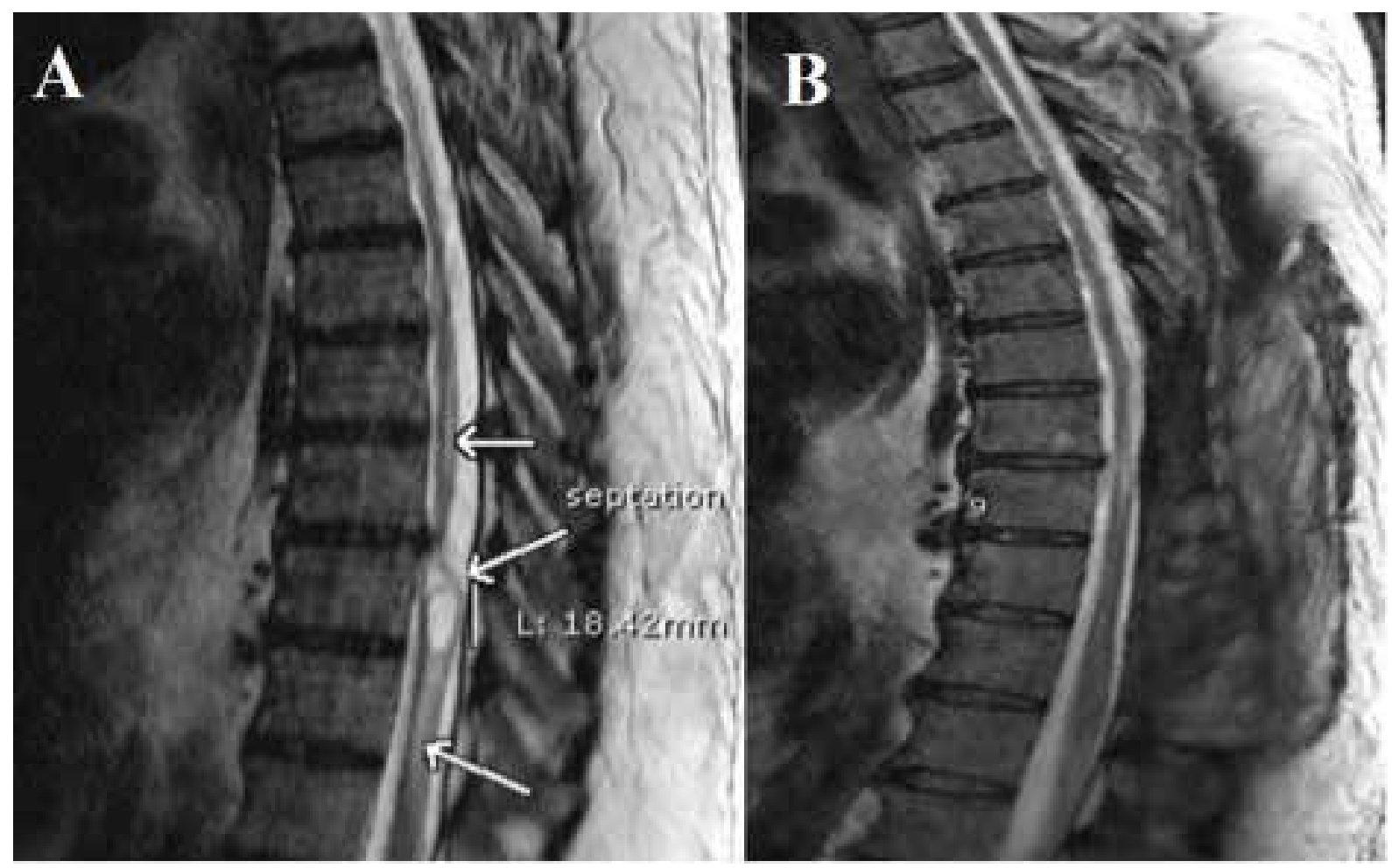

Fig. 1. A) Thoracic MRI demonstrating non-enhancing, expansile, intramedullary, intradural, cystic, $\mathrm{T} 2$ bright cord lesion at the level of T9, with associated syrinx, and cord edema above and below the lesion. B) Resolution of intramedullary cyst following wide resection of arachnoid tissue and fenestration of arachnoid cyst. 
Case 2. The patient is a 56-year-old male presenting with severe low back pain radiating into the bilateral lower extremities, specifically in the anterior aspect of the left lower extremity in an L4 distribution. There was no reported abdominal pain, thoracic radicular pain, or numbness, but there was reported bilateral lower extremity numbness. Lumbar MRI revealed L3-4 disc bulge without stenosis, L4-5 disc bulge with moderate left neural foraminal stenosis without central stenosis, and L5-S1 disc bulge with mild left neural foraminal stenosis without central stenosis (Fig. 2A). Electrodiagnostic studies revealed chronic, mild, left L3, L4, and bilateral S1 radiculopathy.

The patient underwent multiple interventional procedures including epidural steroid injections in conjunction with trials of tramadol, naproxen, and duloxetine, all of which failed to significantly ameliorate symptoms. He also underwent unsuccessful trials of physical therapy, including both aquatic and land-based treatments.

With continued complaints of back and bilateral lower extremity pain and numbness, the patient underwent an SCS trial. Standard percutaneous trial leads were introduced at T12-L1, and the electrodes were threaded to the inferior endplate of the T9 vertebral body (Fig. 2B and $\mathrm{C}$ ). The patient reported greater than $50 \%$ pain relief after his trial as well as improvement in baseline function. He continued to endorse right knee and left ankle pain from prior traumatic injuries. He elected to proceed with placement of a permanent SCS and was referred to neurosurgery for placement of a paddle electrode.

The patient met with the neurosurgeon to discuss implanting a permanent paddle electrode. As part of the preoperative planning, a thoracic MRI was obtained, which revealed an intradural extramedullary mildly T2 hyperintense, T1 isointense mass measuring 2.7 $\times 1.5 \times 1.0 \mathrm{~cm}$ at the level of T9-T10, which resulted in severe central canal stenosis (Fig. 2D).

The patient was referred to a neurosurgeon specializing in spinal cord tumors for surgical excision of the lesion. The patient underwent a thoracic laminectomy, using a posterior approach for resection of the intradural extramedullary spinal cord tumor, and duraplasty. He reported resolution of the majority of the numbness he was experiencing throughout his legs, with the exception of numbness in his heels, within 2 days after the surgery. His previous limitations

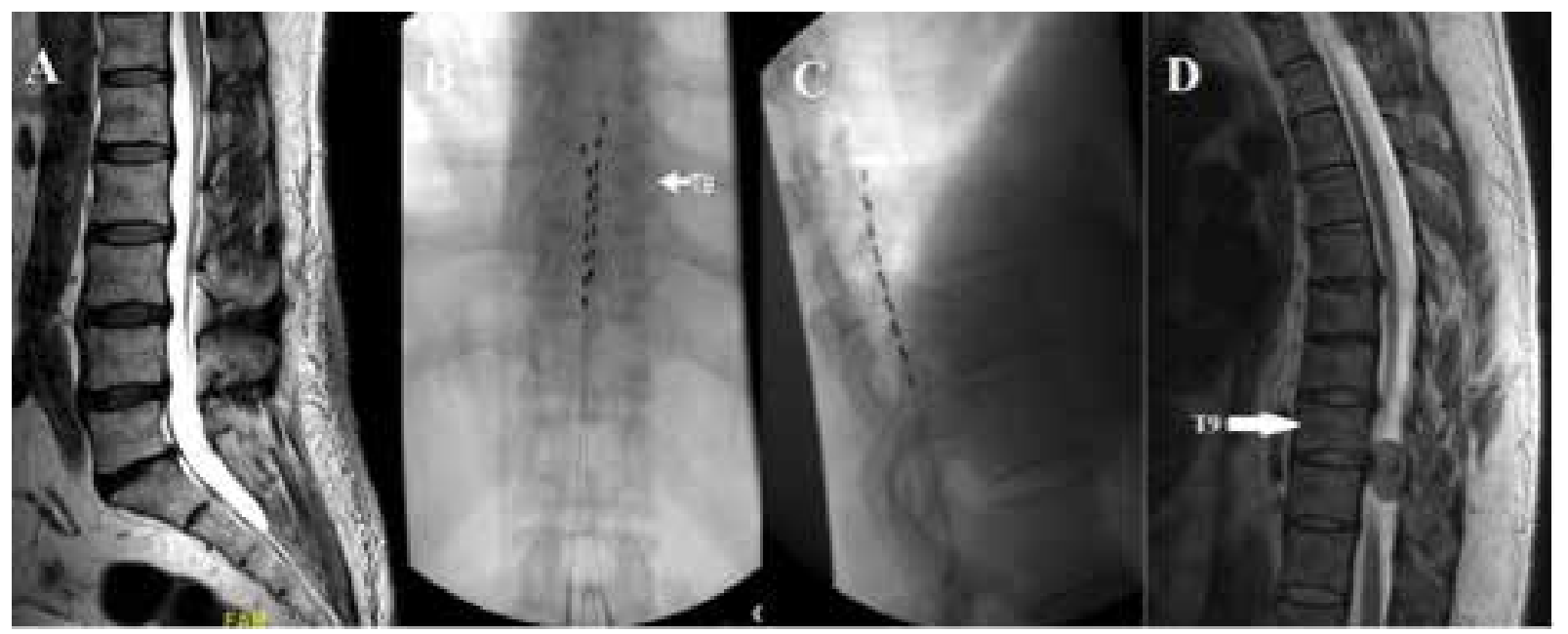

Fig. 2. A) Lumbar MRI demonstrating L3-4 disc bulge without stenosis, L4-5 disc bulge with moderate left neural foraminal stenosis without central stenosis, and L5-S1 disc bulge with mild left neural foraminal stenosis without central stenosis. B and C) Introduction of standard percutaneous trial leads at T12-L1, and threading of electrodes to the inferior endplate of the T9 vertebral body. D) Thoracic MRI revealing an intradural extramedullary mildly T2 hyperintense, $\mathrm{T} 1$ isointense mass measuring $2.7 \times 1.5 \times 1.0 \mathrm{~cm}$ at the level of T9-T10. 
Preoperative Surveillance MRI Prior to Spinal Cord Stimulation

with ambulation have also resolved and participation in a walking program has been initiated.

\section{DISCUSSION}

To date, there have been no guidelines or consensus statements regarding the use of advanced imaging studies of the neuraxis as a screening tool prior to patients undergoing spinal cord stimulation (2). The incidental anatomical lesions detected from routine preprocedural MRI evaluation in these cases illustrate the potential value of imaging prior to SCS electrode placement. In particular, use of thoracic MRI identified arachnoid cyst and meningioma, both with spinal cord compression, which were successfully treated with surgery leading to resolution of a component of the patient's pain and dysfunction in Case 1 and all of the patient's pain and dysfunction in Case 2.

Several important lessons were learned from these cases: 1) The patients' symptoms of pain and weakness at initial evaluation for SCS, initially thought to be secondary to diagnoses of post-laminectomy syndrome and lumbar radiculopathy, were in part or wholly attributable to thoracic arachnoid cyst with spinal cord compression in the first case, and a meningioma with spinal cord compression in the second case. Furthermore, the first patient's initial presentation may also have been secondary to thoracic lesion, obviating the need for 2 subsequent lumbar surgeries and related sequelae; (2) SCS trial was performed in Case 2 prior to thoracic imaging. The thoracic mass was diagnosed prior to surgical paddle electrode placement. This case underscored the importance of obtaining thoracic imaging prior to trial SCS placement and changed the practice of our entire team; (3) Had spinal cord stimulation therapy been undertaken without prior MRI of the thoracic spine, work-up and detection of the thoracic lesions may have been hampered by the limitation in imaging options, potentially delaying treatment and jeopardizing the patient's overall recovery and functional capacity; (4) Spinal cord stimulators and other spinal cord stimulation procedures should only be pursued after very thorough assessment, which includes an appropriate history and physical exam. An astute diagnostician should be cognizant of symptoms and findings not fully explained by the working diagnosis, which in these cases may have increased suspicion for an incidental thoracic lesion on patients' initial presentations.

Although manufacturers of spinal cord stimulator equipment have achieved or are hoping to achieve MRI-compatible systems, this is not yet universal, and when applicable, can be only limited/conditional $(4,5)$. Although CT with myelogram could have been used to diagnose thoracic lesions after SCS implant in patients, most surgeons would agree that MRI would have been the imaging modality of choice (6). Currently, limited screening studies, such as T2 sagittal and axial screening of the cervical or thoracic spine prior to SCS therapies, are not part of the "standard of care" in the work-up of patients undergoing spinal cord stimulation. As "standard of care" has medico-legal implications, with deviation from the standard suggesting "negligence," it may be prudent to consider surveillance imaging as part and parcel of an acute pain physician's diagnostic workup of a patient prior to thoracic spinal cord stimulator treatment (7). These imaging studies would help to rule out anatomic abnormalities or lesions, which may impact the safety of SCS placement, including, but not limited to disc extrusion, spinal canal or neuroforaminal stenosis, tumor, or vascular malformation. For example, thoracic spinal stenosis may lead to difficulties with advancement and accurate placement of percutaneous electrodes (8).

Some may suggest that the value of surveillance imaging studies is limited by their contribution to escalating health care expenditures. Although initial acquisition costs associated with SCS are high, numerous studies have shown that SCS is cost-effective and that these early costs are consistently offset by a reduction in use of health care resources, including drug therapy, physician visits, and hospitalizations (9). In the context of chronic back pain, which in and of itself places a tremendous economic burden on health care, SCS has been shown to be more cost-effective than both conventional medical management (CMM) and surgical reoperation (10). Although preoperative imaging may add to initial increased expenses, longterm benefits may still be achieved, as SCS becomes cost-effective within the first 2 years of treatment and is both clinically superior and cost-saving over a patient's lifetime compared to CMM (11). Finally, the average Medicare cost of performing SCS explanta- 
tion is estimated to be $\$ 5,102$, in addition to a mean cost of $\$ 13,452$ for the initial SCS implant procedure itself (excluding all equipment expenses) (12). In contrast, costs for pre-implant imaging were $\$ 728$ for CT (average of 1.7 scans per patient) and $\$ 688$ for MRI (1.1 scans per patient) (12). While radiologic screening alone cannot be directly linked to subsequent rates of complication requiring explant, these costs illustrate the extensive expenses associated with device implantation and removal. This underscores the value of a thorough evaluation process and supports the use of imaging surveillance protocols to improve screening for optimal patient candidates.

In addition, these cases highlight several issues: 1) If imaging studies at baseline presentation of pain had been performed as part of a more exhaustive initial clinical assessment, thoracic lesions would have been detected much earlier. Subsequently, potentially unnecessary surgeries in the lumbar spine, as well as costs related to loss of productivity and health care expenses, could have been avoided. Ironically, in this case, even the cost of SCS therapy, which one may posit was indicated as the result of spinal intervention and creation of scar tissue, may have been avoided as well; 2) Before these cases arose, there was inequity in our practice, with some interventionists obtaining surveillance imaging while some were not. Interestingly, the neurosurgeon in our practice always reviewed advanced imaging prior to surgical paddle electrode placement. This discrepancy highlighted the need for consistency in our practice and raised the bar for all providers to obtain surveillance imaging; 3) Although these cases focused on thoracic procedures, the utility of advanced imaging would certainly apply to cervical spinal cord procedures as well. In fact, given the smaller diameter of the spinal canal in the cervical spine, any potential undetected spaceoccupying lesion in that region could lead to more disastrous consequences if a cervical percutaneous or paddle lead were to be placed (13).

\section{CONCLUSION}

Advanced imaging (including MRI or CT with or without myelogram, if MRI is contraindicated) should be considered as part of the comprehensive work-up for patients undergoing SCS in the thoracic or cervical spine. The added benefits of radiologic screening, including identification of critical structural abnormalities or conditions that can be surgically addressed prior to or instead of SCS therapy, outweigh the short-term costs and minimal risks. Given that poor patient selection is one of the most challenging aspects of SCS and is associated with poorer outcomes $(14,15)$, practitioners performing spinal cord stimulation should consider adopting a local preoperative imaging protocol for reasons of best practice. These 2 cases underscore the age-old lesson that interventional pain practitioners, and in reality any thorough physician, should pay close attention to an individualized patient's clinical scenario and look further if the diagnosis does not explain the signs and symptoms at hand. 
Preoperative Surveillance MRI Prior to Spinal Cord Stimulation

\section{REFERENCES}

1. Wolter T. Spinal cord stimulation for neuropathic pain: Current perspectives. J Pain Res 2014; 7:651-663.

2. Walsh KM, Machado AG, Krishnaney AA. Spinal cord stimulation: A review of the safety literature and proposal for perioperative evaluation and management. Spine ] 2015; 15:1864-1869.

3. Chincholkar M, Eldabe S, Strachan R, Brookes M, Garner F, Chadwick R, Gulve A, Ness J. Prospective analysis of the trial period for spinal cord stimulation treatment for chronic pain. Neuromodulation 2011; 14:523-529.

4. Desai MJ, Hargens LM, Breitenfeldt MD, Doth AH, Ryan MP Gunnarsson C, Safriel Y. The rate of magnetic resonance imaging in patients with spinal cord stimulation. Spine 2015; 40:E531E537.

5. Rubino S, Adepoju A, Kumar V, Prusik J, Murphy N, OwusuSarpong S, Pilitsis JG. MRI conditionality in patients with spinal cord stimulation devices. Stereotact Funct Neurosurg 2016; 94:254-258.

6. Shah LM, Salzman KL. Imaging of spinal metastatic disease. Int J Surg Oncol 2011; 2011:769753.

7. Proehl JA, Hoyt KS. Evidence versus standard versus best practice: Show me the data! Adv Emerg Nurs ] 2012; 34:1-2.

8. Ghaly RF, Lissounov A, Candido KD, Knezevic NN. Are there guidelines for implantable spinal cord stimulator therapy in patients using chronic anticoagulation therapy? - A review of deci- sion-making in the high-risk patient. Surg Neurol Int 2016; 7:33.

9. Taylor RS, Taylor RJ, Van Buyten J-P, Buchser E, North R, Bayliss $S$. The cost effectiveness of spinal cord stimulation in the treatment of pain: A systematic review of the literature. J Pain Symptom Manage 2004; 27:370-378.

10. Hoelscher C, Riley J, Wu C, Sharan A. Cost-effectiveness data regarding spinal cord stimulation for low back pain. Spine 2017; 42:S72-S79.

11. Taylor RJ, Taylor RS. Spinal cord stimulation for failed back surgery syndrome: A decision-analytic model and cost-effectiveness analysis. Int J Technol Assess Health Care 2005; 21:351-358.

12. Kumar K, Bishop S. Financial impact of spinal cord stimulation on the healthcare budget: A comparative analysis of costs in Canada and the United States. J Neurosurg Spine 2009; 10:564573.

13. Campbell PG, Falowski SM. Epidural paddle placement for spinal cord stimulation. In: Falowski SM, Pope JE (eds). Integrating Pain Treatment into Your Spine Practice. New York, Springer International Publishing, 2016: pp. 165-173.

14. Villavicencio AT, Burneikiene S. Elements of the pre-operative workup, case examples. Pain Med 2006; 7:S35-S46.

15. Cameron T. Safety and efficacy of spinal cord stimulation for the treatment of chronic pain: A 20-year literature review. J Neurosurg 2004; 100:254-267. 
